INSTITUTE OF APPLIED MECHANICS, RUSSIAN ACADEMY OF SCIENCES, MOSCOW, RUSSIA

UDC 579 872: 579 222.2, LBC 28.072

03 - life sciences

\title{
OXIDATION OF GERANYL-N-PHENYLCARBAMATE BY FUNGUS BEAUVERIA BASSIANA WITH AIM TO OBTANING OF NEW ANTI-CANCER DRUGS
}

\section{Sc. D. I. A. Parshikov}

Abstract. The microbial oxidation of geranyl- $N$-phenylcarbamate by fungus Beauveria bassiana was investigated. Oxidation of the $\mathrm{C} 3-\mathrm{C} 4$ double bond of the parent molecule leads to regioselective formation of $O$-3,4-epoxyheranyl- $N$-phenylcarbamate in $30 \%$ yield.

Keywords: monoterpenes, fungi, Beauveria bassiana, oxidation

\section{ИНСТИТУТ ПРИКЛАДНОЙ МЕХАНИКИ РАН, МОСКВА, РОССИЯ}

УДК 579 872: 579 222.2, ББК 28.072

03 - биологические науки

\section{ОКИСЛЕНИЕ ГЕРАНИЛ- $N$-ФЕНИЛКАРБАМАТА ГРИБОМ ВЕАUVERIA ВASSIANA С ЦЕЛЬЮ ПОЛУЧЕНИЯ НОВЫХ АНТИРАКОВЫХ ПРЕПАРАТОВ}

\section{Доктор биологических наук И. А. Паршиков}

Реферат. Было исследовано микробное окисление геранил- $N$-фенилкарбамата грибом Beauveria bassiana. Окисление двойной связи С3 - С4 исходной молекулы приводит к реги-

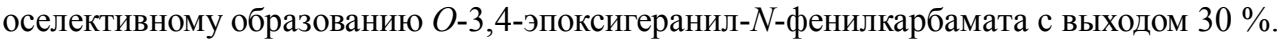

Ключевые слова: монотерпены, грибы, Beauveria bassiana, окисление

\section{Введение}

Производные монотерпенов используются для получения душистых веществ, пестицидов, аттрактантов. Накопленные в последнее время научные данные показали, что монотерпены выделенные из растений проявляют противораковую активность, в частности, гераниол и его производные [1]. Ранее установлено, что некоторые микроорганизмы. способны окислять кратные связи монотерпенов, однако, большинство процессов шли нерегиоселективно и приводили образованию целого спектра окисленных продуктов [2]. При попытках микробного окисления нерола, цитраля и гераниола штаммом Aspergillus niger была обнаружена смесь многочисленных продуктов [2].

Мы изучили возможность окисления геранил- $N$-фенилкарбамата грибом Beauveria bassiana BКМ F-2533 (рис. 1), с целью получения новых производных с потенциально возможными противоопухолевыми свойствами.

\section{Материалы и методы исследования}

Штамм гриба Beauveria bassiana ВКМ F-2533 был получен из Всероссийской коллекции микроорганизмов. Приготовление посевного материала, условия инкубации и экстракции продуктов описаны нами ранее [3]. Субстрат для трансформации (1) добавляли в концентрации 50 мг/л.

Продукты выделяли из смеси методом колоночной хроматографии на силикагеле Kieselgel 0.036-0.200 (Merck, ФРГ). Хроматографию в тонком слое проводили на пластинках с силикагелем Kieselgel 60 F254 (Merk, ФРГ) в системе растворителей: этилацетат гексан - метанол $(5: 5: 1)$. 
Выделенные вещества идентифицировали на основании масс-спектров и спектров протонного магнитного резонанса ( ${ }^{1} \mathrm{H}$ ЯМР).

Спектры ${ }^{1} \mathrm{H}$ ЯMP снимали в растворе $\mathrm{CDCl}_{3}$ на приборе Brucker DPX 300 (Brucker, CША). Macc-спектры записывали на приборе Finnigan TSQ 700 (Finnigan Corp., США).

\section{Результаты исследования и их обсуждение}

При трансформации геранил- $N$-фенилкарбамата (1) реакция шла региоселективно и нами был получен единственный продукт O-3,4-эпоксигеранил- $N$-фенилкарбамат (2) с молекулярным ионом при $m / z 289, \mathrm{R}_{\mathrm{f}}=0,62$ и выходом $30 \%$ (рис. 1 ).

Масс-спектр соединения 2: 289 (25), 205 (10), 170 (6), 152 (28), 137(30), 119 (21), 93 (100), 91 (22), 77 (27), 67 (21), 58 (20), 43 (35).

Образование ионов $\mathrm{m} / \mathrm{z} 205$, и особенно 58 подтверждало оксирановый характер продукта окисления [4].

Спектр ${ }^{1} \mathrm{H}$ ЯМР - 1,15 (с, 3H, 2- $\left.\mathrm{CH}_{3}\right) ; 1,20$ (с, 3H, 1- $\left.\mathrm{CH}_{3}\right) ; 1,34-1,69$ (м, 2H, 5- $\left.\mathrm{CH}_{2}\right), 1,74$ (с, $3 \mathrm{H}, 8-\mathrm{CH}_{3}$ ), 2,08-2,37 (м, 2H, 6- $\mathrm{CH}_{2}$ ), 3,34 (д, 1H, 4-CH), 4,67 (д, 2H, 10- $\mathrm{CH}_{2}$ ), 5,38 (д, 1Н, 9$\mathrm{CH}), 6,69(\mathrm{M}, 1 \mathrm{H}, \mathrm{NH}), 7,04-7,45\left(\mathrm{M}, 5 \mathrm{H}, \mathrm{C}_{6} \mathrm{H}_{5}\right)$.

Ранее были описаны процессы трансформации геранил- $N$-фенилкарбамата (1) с использованием штамма $A$. niger LCP 521, что привело к образованию диола при окислении двойной связи С3 - C4, а также к сопутствующей смеси многочисленных продуктов с их ничтожным выходом [5].

Многие противоопухолевые препараты, используемые в настоящее время в клинической практике, были выделены из различных видов растений или синтезированы на основе таких веществ $[1,6]$. Мы предлагаем способ региоселективного получения $O-3,4$-эпоксигеранил- $N$-фенилкарбамата, как потенциального кандидата для создания новых противоопухолевых лекарств.
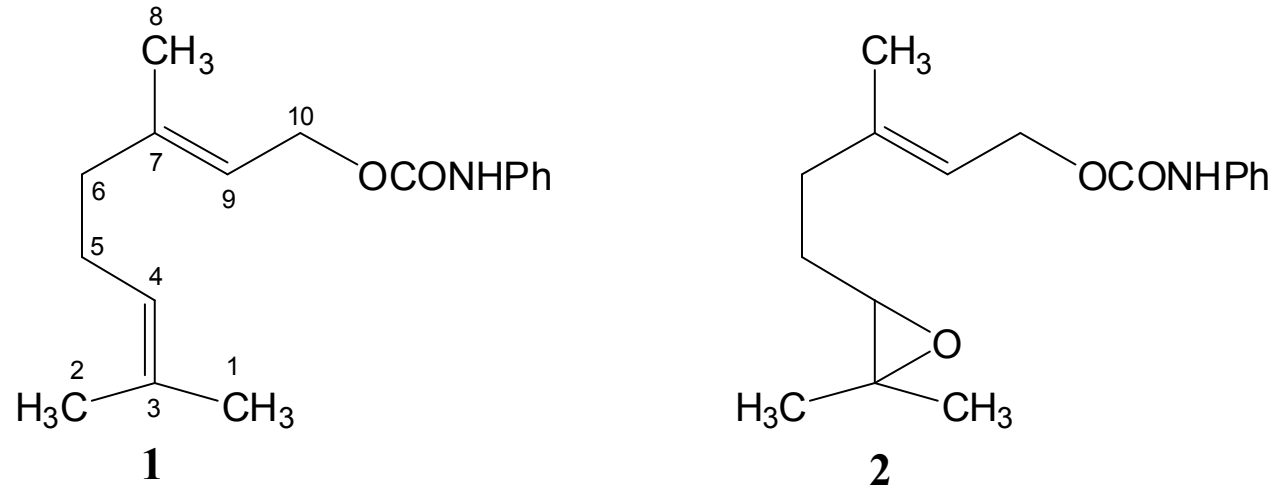

Рис. 1. Микробное окисление геранил- $N$-фенилкарбамата (1) с образованием $O-3,4$-эпоксигеранил- $N$-фенилкарбамата (2).

\section{ПРИМЕЧАНИЯ}

1. Sobral M. V., Xavier A. L., Lima T. C., Sousa D. P. Antitumor Activity of Monoterpenes Found in Essential Oils // The Scientific World Journal. 2014. V.2014. Article ID 953451, 35 p. http://dx.doi.org/10.1155/2014/953451

2. Demyttenaere JC, del Carmen Herrera M, De Kimpe N. Biotransformation of geraniol, nerol and citral by sporulated surface cultures of Aspergillus niger and Penicillium sp. // Phytochemistry. 2000. V. 55. P. 363-373. 
3. Parshikov I. A., Hernandes-Luna C. E., Zaraisky E. I. Microbial transformation of the antimalarial and anticancer drug artemisinin by white-rot basidiomycetes // Asian Journal of Microbiology, Biotechnology and Environmental Sciences. 2018. V. 20. No. 4. P. 1093-1096.

4. Elgendya E. M., Khayyat S. A. Oxidation Studies on some natural monoterpenes: citral, pulegone, and camphene // Russian Journal of Organic Chemistry. 2008. V. 44. No. 44. P. 10704280.

5. Fourneron J. D., Archelas A., Furstoss R. Microbial transformations. 12. Regiospecific and asymmetric oxidation of the remote double bond of geraniol // Journal of Organic Chemistry. 1989. V. 54. P. 4686-4689.

6. Parshikov I. A. Microorganisms in Chemistry of Terpenoids. - Dallas: Primedia E-launch LLC, 2016. - $102 \mathrm{p}$.

\section{REFERENCES}

1. Sobral M. V., Xavier A. L., Lima T. C., Sousa D. P. Antitumor Activity of Monoterpenes Found in Essential Oils // The Scientific World Journal. 2014. V. 2014. Article ID 953451, 35 p. http://dx.doi.org/10.1155/2014/953451

2. Demyttenaere JC, del Carmen Herrera M., De Kimpe N. Biotransformation of geraniol, nerol and citral by sporulated surface cultures of Aspergillus niger and Penicillium sp. // Phytochemistry. 2000. V. 55. P. 363-373.

3. Parshikov I. A., Hernandes-Luna C. E., Zaraisky E. I. Microbial transformation of the antimalarial and anticancer drug artemisinin by white-rot basidiomycetes // Asian Journal of Microbiology, Biotechnology and Environmental Sciences. 2018. V. 20. No. 4. P. 1093-1096.

4. Elgendya E. M., Khayyat S. A. Oxidation Studies on some natural monoterpenes: citral, pulegone, and camphene // Russian Journal of Organic Chemistry. 2008. V. 44. N 44. P. 10704280 .

5. Fourneron J. D., Archelas A., Furstoss R. Microbial transformations. 12. Regiospecific and asymmetric oxidation of the remote double bond of geraniol. // Journal of Organic Chemistry. 1989. V. 54. P. 4686-4689.

6. Parshikov I. A. Microorganisms in Chemistry of Terpenoids. - Dallas: Primedia E-launch LLC, 2016. - $102 \mathrm{p}$. 